\title{
Genetic Mutations and Mitochondrial Toxins Shed New Light on the Pathogenesis of Parkinson's Disease
}

\author{
Shigeto Sato and Nobutaka Hattori \\ Department of Neurology, Juntendo University School of Medicine, Tokyo 113-8421, Japan \\ Correspondence should be addressed to Nobutaka Hattori, nhattori@juntendo.ac.jp \\ Received 11 April 2011; Revised 2 June 2011; Accepted 12 June 2011 \\ Academic Editor: Honglei Chen
}

Copyright (๑) 2011 S. Sato and N. Hattori. This is an open access article distributed under the Creative Commons Attribution License, which permits unrestricted use, distribution, and reproduction in any medium, provided the original work is properly cited.

\begin{abstract}
The cellular abnormalities in Parkinson's disease (PD) include mitochondrial dysfunction and oxidative damage, which are probably induced by both genetic predisposition and environmental factors. Mitochondrial dysfunction has long been implicated in the pathogenesis of PD. The recent discovery of genes associated with the etiology of familial PD has emphasized the role of mitochondrial dysfunction in PD. The discovery and increasing knowledge of the function of PINK1 and parkin, which are associated with the mitochondria, have also enhanced the understanding of cellular functions. The PINK1-parkin pathway is associated with quality control of the mitochondria, as determined in cultured cells treated with the mitochondrial uncoupler carbonyl cyanide m-chlorophenylhydrazone (CCCP), which causes mitochondrial depolarization. To date, the use of mitochondrial toxins, for example, 1-methyl-4-phynyl-tetrahydropyridine (MPTP) and CCCP, has contributed to our understanding of PD. We review how these toxins and familial PD gene products are associated with and have enhanced our understanding of the role of mitochondrial dysfunction in PD.
\end{abstract}

\section{Introduction}

Parkinson's disease (PD) is the most common neurodegenerative movement disorder, affecting $1 \%$ of the population above the age of 60 . The classical form of the disease is characterized clinically by rigidity, resting tremor, bradykinesia, and postural instability. In addition to these four cardinal symptoms, many nonmotor symptoms frequently appear in $\mathrm{PD}$, such as cognitive impairment, hallucinations, delusion, behavioral abnormalities, depression, disturbances of sleep and wakefulness, loss of smell, pain, and autonomic dysfunctions such as constipation, hypotension, urinary frequency, impotence, and sweating. The pathological hallmarks of PD are the preferential loss of dopaminergic neurons of the substantia nigra (SN) pars compacta and formation of Lewy bodies. Exposure to environmental factors inducing mitochondrial toxin like1-methyl-4-phynyl-tetrahydropyridine (MPTP) produces selective degeneration of dopaminergic neurons in SN and results in an irreversible Parkinsonism [1-3]. The active metabolite of MPTP, 1-methyl-4-phenylpyridinium ion (MPP+), is an inhibitor of complex I, and it accumulates in dopaminergic neurons because it is actively transported via dopamine transporter (DAT) [4-6]. The inhibition of the electron transport induces oxidative damage by increasing the formation of reactive oxygen species (ROS) and leads to further mitochondrial dysfunction [7]. These findings were supported by evidence of oxidative damage including an increase in lipid peroxide [8], decrease in glutathione [9], increase in hydroxynonenal-modified proteins [10], and increase in 8-hydroxy-deoxy guanine [11] in SN. ROS impair mitochondrial proteins, further aggravating mitochondrial function. Ultimate outcomes are dissipation of mitochondrial membrane potential and the release of cytochrome $\mathrm{c}$ into the cytoplasm and activation of the apoptotic cascade. A biochemical link between MPTP toxicity and Parkinsonism was confirmed with the finding of low levels of complex I in the SN, skeletal muscle, and platelets in patients with PD $[12,13]$. In contrast, it remains unknown whether this systemic deficiency of complex I is crucially related to dopaminergic cell loss in PD. Rats administered rotenone (an inhibitor of complex I) developed neuronal degeneration and formation of synuclein-positive 
inclusions; however, the degree of complex I inhibition was not severe enough to induce brain mitochondrial dysfunction [14]. Although inhibition of complex I and production of free radical result in increased oxidative stress, it remains unclear whether such dysfunction is a primary or a secondary process in the pathogenesis of the disease.

\section{Involvement of Two Mitochondrial Toxic Pathways in Synuclein, DJ-1, and Parkin Mice Model}

Several mutations of the synuclein gene (SNCA) at the PARK1 locus induce autosomal dominant Parkinsonism. Three missense mutations: A53T [15], A30P [16], and E46K [17], duplications [18-21], and triplications [22, 23] of SNCA have so far been described. Triplications are associated with Parkinsonism and dementia, and the age of onset is younger than the other mutations, and the neuropathological changes are those of diffuse Lewy body disease. Regarding the pathogenesis of PARK1-linked $\mathrm{PD}$, accumulation of normal synuclein is likely to predispose nigral neurons for protofibril formation. Toxicity associated with increased synuclein expression is an important cellular event that enhances the genetic predisposition to sporadic PD. At present, indirect evidence suggests a relationship between synuclein and oxidative stress, including protein carbonylation and lipid peroxidation. Furthermore, synuclein-deficient mice were found to have striking resistance to MPTP-induced degeneration of dopaminergic neurons, and this resistance appeared to be related to failure of the toxin itself. Interestingly, there was dissociation in the resistance between MPTP- and rotenoneinduced cell vulnerability of synuclein-null dopaminergic neurons [24]. This result suggests that MPTP associates with synuclein through another pathway independent of complex I inhibition (mitochondrial dysfunction), to finally induce dopaminergic cell death. Several mutations of the DJ-1 gene at the PARK7 locus induce autosomal recessive Parkinsonism [25]. Clinical phenotype is characterized by an onset in the midthirties, good levodopa response, and slow disease progression. Several lines of evidence suggest that it plays a role in the oxidative stress response [26, 27]. Subcellular localization studies have shown DJ-1 to be present in the cytosol, mitochondria, and nucleus [26, 28, 29]. Junn et al. [30] showed that in response to oxidative stress, some of the DJ-1 protein is translocated from its major cytosolic pool to mitochondria and nucleus. DJ-1 null mice are vulnerable to MPTP [31]. On the other hand, Thomas et al. [32] reported that the susceptibility of SN to MPTP in mice is independent of parkin activity. In short, the absence of parkin does not seem to increase the vulnerability of dopaminergic neurons to MPTP intoxication. Another study also found that oxidative stress, including MPTP, altered parkin solubility, causing parkin aggregation, thereby suggesting parkin dysfunction as a pathogenic mechanism of sporadic PD [33].

\section{Functional Interplay between PINK1 and Parkin to Maintain Mitochondrial Integrity}

Many mutations of the parkin gene at the PARK2 locus induce autosomal recessive Parkinsonism [34-38]. The usual age of onset is between 20 and 40 years. Clinical features consist of dystonia and sleep benefit, which are also characteristic symptoms. Despite affected patients responding well to levodopa, they soon develop motor fluctuations. Conversely, mutations of the PINK1 (PTEN-induced kinase 1) gene at the PARK6 locus induce autosomal recessive Parkinsonism. The age of onset is slightly delayed relative to PARK2, that is, from 32 to 48 years [39]. The affected patients show levodopa-responsive Parkinsonism. PINK1 contains an $\mathrm{N}$-terminal mitochondrial targeting signal and a highly conserved serine/threonine kinase domain, and many missense and nonsense mutations have been reported at the kinase domain [40-44]. In particular, the identification of PINK1 mutations has strongly implicated mitochondrial dysfunction in the pathogenesis of PD [40]. The activity of PINK1 kinase is crucial for mitochondrial maintenance via TRAP phosphorylation [45]. The loss of PINK1 function results in increased vulnerability to various stresses [4648]. Drosophila models have demonstrated that PINK1 and parkin ensure stable mitochondrial function. Parkin null mutants show severe mitochondrial pathology associated with reduced lifespan, apoptosis, and muscle degeneration [49]. While the PINK1 mutant phenotype can be rescued by parkin gene overexpression $[50,51]$, the converse does not occur, suggesting that parkin acts downstream of PINK1 in a common pathway to maintain mitochondrial integrity. PINK1 loss-of-function results in reduced mitochondrial membrane potential [52], and the PINK1-parkin pathway is associated with mitochondrial elimination in cultured cells treated with the mitochondrial uncoupler carbonyl cyanide m-chlorophenylhydrazone (CCCP), which causes mitochondrial depolarization [53-58]. The exact mechanism underlying CCCP-induced mitochondrial depolarization, leading to mitochondrial autophagy, has been examined in detail. At steady state, parkin is localized throughout the cytosol but not in the mitochondria. However, parkin was rapidly recruited into the mitochondria when HeLa cells were treated with CCCP [55]. Furthermore, PINK1 recruits parkin from the cytoplasm to the low-membrane potential mitochondria, resulting in the mitochondrial degradation. Interestingly, the ubiquitin-ligase activity of parkin is repressed in the cytoplasm at steadystate; however, PINK1-dependent mitochondrial localization triggered by mitochondrial depolarization liberates the potential enzymatic activity of parkin. While CCCP is well described, its mitochondrial toxic effects provide new insights on the functional interplay between PINK1 and parkin.

\section{Accumulation of PINK1 in Damaged Mitochondria}

PINK1 is localized in both the mitochondria $[40,59]$ and the cytoplasm $[55,60]$. Treatment with CCCP results in gradual accumulation of PINK1 and translocation of the cytoplasmic 


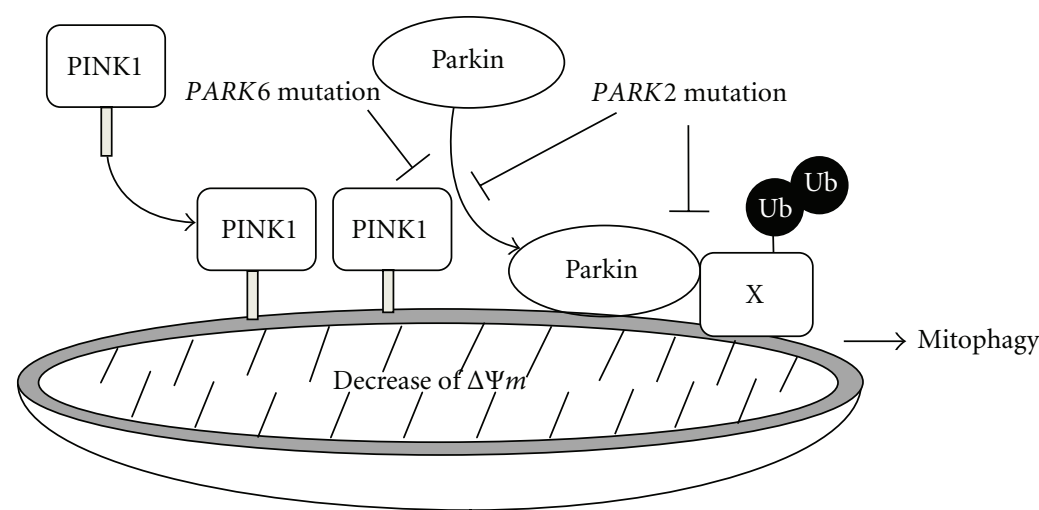

FIGURE 1: Schematic representation of PINK1-parkin-mediated mitophagy. In damaged mitochondria, PINK1 and parkin regulate mitochondrial elimination by inducing mitophagy. Under steady state, PINK1 is cleaved and degraded rapidly in the mitochondria. This process may be inhibited by the mitochondrial depolarization, resulting in PINK1 accumulation in the mitochondria. This accumulation is a crucial signal for parkin recruitment to the mitochondria. Parkin is presumed to ubiquitinate substrate (X), resulting in the induction of mitophagy.

PINK1 to the mitochondria. The subcellular localization of PINK1 is regulated by the mitochondrial membrane potential. Such accumulation may be the first trigger of PINK1-related parkin recruitment. Co-overexpression of PINK1 and parkin results in their colocalization in the mitochondria [61]. Even when these cells were not treated with CCCP, overexpression of PINK1 was associated with translocation of parkin to the cells, together with their mitochondrial aggregation.

Moreover, overexpression of both PINK1 and parkin in the cells resulted in the complete disappearance of the mitochondria. These results suggest that both PINK1 and parkin are indispensable for mitochondrial elimination and that accumulation of PINK1 in the mitochondria results in recruitment of parkin to the mitochondria even in the absence of CCCP [54].

\section{PINK1 Kinase Activity Is Essential for Translocation of Parkin}

PINK1 is composed of an atypical N-terminal mitochondrial targeting signal and transmembrane domain, kinase domain in the middle, and a conserved C-terminal domain, and deletion of the N-terminal amino acids abolished the mitochondrial localization of PINK1 [62]. Among other mutations, G309D, L347P, and G409V are associated with reduction in PINK1-kinase activity, and a C-terminal domain deletion mutant is associated with PINK1 dysfunction [63, 64]. The G309D/L347P/G409V mutants preserved mitochondrial localization, though their mitochondrial elimination was less compared to cells expressing both the wild-type PINK1 and parkin. When introduced into PINK1-deficient cells, the mutants were unable to complement the localization of parkin [55]. These results indicate that targeting the kinase activity and mitochondrial distribution of PINK1 is important for the mitochondrial recruitment of parkin (Figure 1).

\section{PINK1 Deficiency Itself Causes Respiratory Chain Defects}

Impaired mitochondrial respiration was observed in the brain of PINK1 null mice [65] although the mechanism linking PINK1 to mitochondrial membrane potential remains to be determined. Amo et al. [66] reported depletion of the mitochondrial membrane potential and cellular ATP levels $(\sim 80 \%)$ in PINK1-deficient mouse embryonic fibroblasts (MEFs) compared with those in littermate wild-type MEFs. However, loss of PINK1 did not alter mitochondrial proton leak, which reduces the membrane potential in the absence of ATP synthesis. Instead, the authors reported reduced activity of the respiratory chain, which produces the membrane potential by oxidizing substrates using oxygen. The $\mathrm{H}_{2} \mathrm{O}_{2}$ production rate by PINK1 null mitochondria was lower due to low oxygen consumption rate, while the proportion $\left(\mathrm{H}_{2} \mathrm{O}_{2}\right.$ production rate per oxygen consumption rate) was higher. These results suggest that mitochondrial dysfunction in PD is not caused by proton leak, but by a defective respiratory chain. Furthermore, rate of free radical leak was significantly higher in PINK1-deficient MEFs than in wildtype MEFs. Because the differences disappeared with the addition of rotenone (inhibitor of complex I, which inhibits reverse electron flow from coenzyme $Q$ to complex I), conceivably ROS generation enhanced by loss of PINK1 was mostly from complex I. With regard to PINK1-related PD, ROS may be an important factor. The above may also explain why cytoplasmic PINK1 protects neurons against MPTP [47]. Inhibition of complex I itself is associated with increased ROS production [67]. These results are at least in part consistent with those of previous studies, suggesting that MPTP and rotenone induce neuronal cell death by inhibiting complex I activity, leading to a PD-like phenotype [68-70] (Figure 2).

It is not doubtful that ROS generation is harmful to the cells, but the process of cell death is supposed to be slow. The crucial point is how inhibition of complex I 


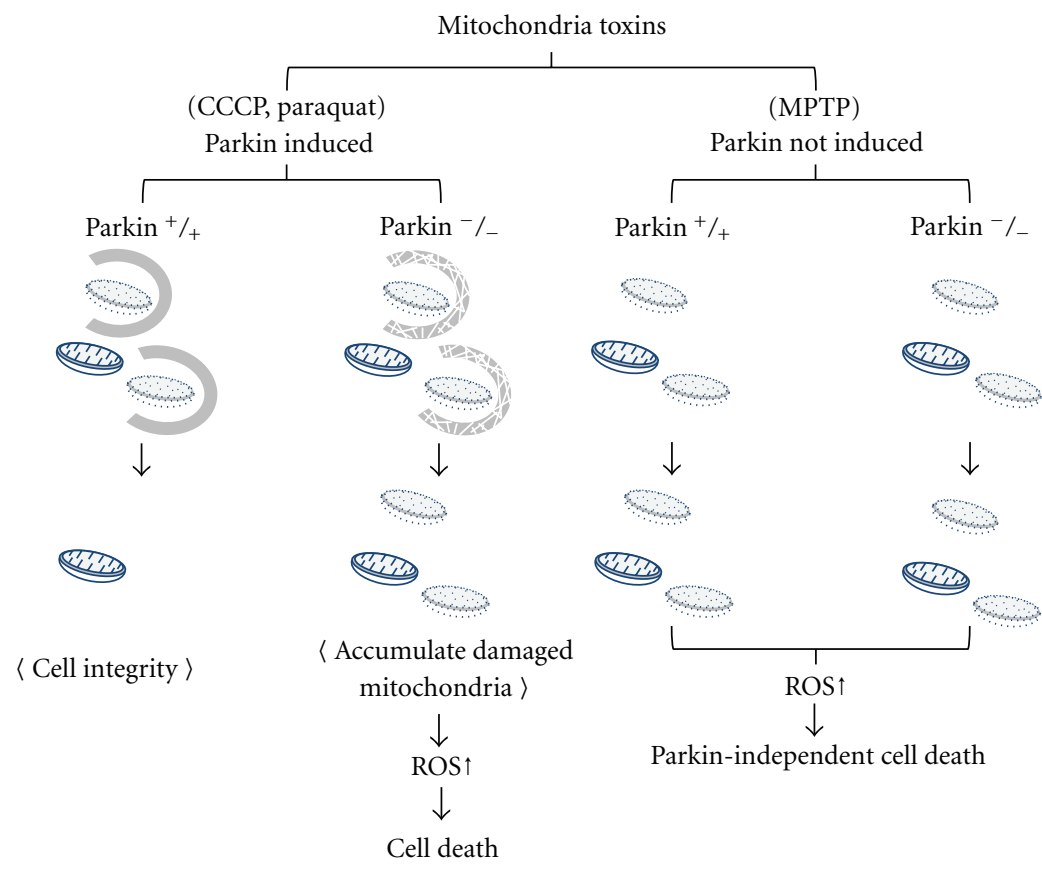

Figure 2: Two mechanisms of mitochondrial toxicity and parkin function. The effect of mitochondrial toxicity is different between CCCP and MPTP. Treatment with CCCP recruits parkin to the mitochondria resulting in mitophagy to keep mitochondrial integrity. Parkin deficiency is associated with accumulation of damaged mitochondria and accelerated cell death. Treatment with MPTP does not necessarily induce parkin. Parkin may be the sensor of damage-adaptive autophagy.

affects mitochondrial dysfunction including mitochondrial depolarization. Considering that the onset of PARK6 (at 32-48 years) is slightly delayed relative to that of PARK2 [39], some cases of PINK1 mutation might not affect parkin recruitment and thus maintain at least part of mitochondrial integrity. This may explain the late onset of PARK6. On the other hand, parkin did not translocate into the mitochondria when cells were treated with MPTP (our unpublished data). This finding means that inhibition of complex I does not necessarily induce low membrane potential. Further research is needed to investigate two independent pathogenic mechanisms related to MPTP and CCCP (Figure 2).

\section{Conclusion}

Cell death of dopaminergic neurons is due to a combination of exogenous stress and genetic predisposition. The discovery of PD genes has provided important insight including an understanding of PINK1-parkin mediated mitophagy. Furthermore, mitochondrial toxins provided crucial clues: (1) CCCP directly affects mitochondrial dysfunction and induces mitophagy; (2) MPTP toxicity seems to alter ROS generation rather than mitochondrial depolarization. The effects of mitochondrial toxins do not seem to be a oneway manner. The information is available for understanding the pathogenesis in PD. Here, we touched on the fringes of molecular mechanisms of PINK1-parkin-mediated mitophagy. Further research will elucidate how this quality control system applies to neurons.

\section{Abbreviations}

CCCP: Carbonyl cyanide m-chlorophenylhydrazone

DAT: Dopamine transporter

MEFs: Mouse embryonic fibroblasts

MPTP: 1-methyl-4-phynyl-tetrahydropyridine

PD: $\quad$ Parkinson's disease

PINK1: PTEN-induced putative kinase1

ROS: Reactive oxygen species

SN: Substantia nigra.

\section{References}

[1] G. C. Davis, A. C. Williams, S. P. Markey et al., "Chronic parkinsonism secondary to intravenous injection of meperidine analogues," Psychiatry Research, vol. 1, no. 3, pp. 249254, 1979.

[2] J. W. Langston, P. Ballard, J. W. Tetrud, and I. Irwin, "Chronic parkinsonism in humans due to a product of meperidineanalog synthesis," Science, vol. 219, no. 4587, pp. 979-980, 1983.

[3] R. S. Burns, C. C. Chiueh, S. Markey, M. H. Ebert, D. Jakobowicz, and I. J. Kopin, "A primate model of Parkinson's disease, selective destruction of substantianigra, pars compactadopaminergic neurons by N-methyl-4-phenyl-1,2,3,6tetrahydropyridine," Proceedings of the National Academy of Sciences of the United States of America, vol. 80, pp. 4546-4550, 1983. 
[4] W. J. Nicklas, I. Vyas, and R. E. Heikkila, "Inhibition of NADH-linked oxidation in brain mitochondria by 1-methyl4-phenyl-pyridine, a metabolite of the neurotoxin, 1-methyl4-phenyl-1,2,5,6-tetrahydropyridine," Life Sciences, vol. 36, no. 26, pp. 2503-2508, 1985.

[5] R. R. Ramsay, J. I. Salach, J. Dadgar, and T. P. Singer, "Inhibition of mitochondrial NADH dehydrogenase by pyridine derivatives and its possible relation to experimental and idiopathic parkinsonism," Biochemical and Biophysical Research Communications, vol. 135, no. 1, pp. 269-275, 1986.

[6] Y. Mizuno, T. Saitoh, and N. Sone, "Inhibition of mitochondrial NADH-ubiquinone oxidoreductase activity by 1 methyl-4-phenylpyridinium ion," Biochemical and Biophysical Research Communications, vol. 143, no. 1, pp. 294-299, 1987.

[7] G. Fiskum, A. Starkov, B. M. Polster, and C. Chinopoulos, "Mitochondrial mechanisms of neural cell death and neuroprotective interventions in Parkinson's disease," Annals of the New York Academy of Sciences, vol. 991, pp. 111-119, 2003.

[8] D. T. Dexter, C. J. Carter, F. R. Wells et al., "Basal lipid peroxidation in substantia nigra is increased in Parkinson's disease," Journal of Neurochemistry, vol. 52, no. 2, pp. 381-389, 1989.

[9] E. Sofic, K. W. Lange, K. Jellinger, and P. Riederer, "Reduced and oxidized glutathione in the substantia nigra of patients with Parkinson's disease," Neuroscience Letters, vol. 142, no. 2, pp. 128-130, 1992.

[10] A. Yoritaka, N. Hattori, K. Uchida, M. Tanaka, E. R. Stadtman, and Y. Mizuno, "Immunohistochemical detection of 4-hydroxynonenal protein adducts in Parkinson disease," Proceedings of the National Academy of Sciences of the United States of America, vol. 93, no. 7, pp. 2696-2701, 1996.

[11] H. Shimura-Miura, N. Hattori, D. Kang, K. I. Miyako, Y. Nakabeppu, and Y. Mizuno, "Increased 8-oxo-dGTPase in the mitochondria of substantia nigral neurons in Parkinson's disease," Annals of Neurology, vol. 46, no. 6, pp. 920-924, 1999.

[12] Y. Mizuno, S. Ohta, M. Tanaka et al., "Deficiencies in complex I subunits of the respiratory chain in Parkinson 's disease," Biochemical and Biophysical Research Communications, vol. 163, pp. 1450-1455, 1989.

[13] A. H. V. Schapira, J. M. Cooper, D. Dexter, P. Jenner, J. B. Clark, and C. D. Marsden, "Mitochondrial complex I deficiency in Parkinson's disease," The Lancet, vol. 1, no. 8649, p. 1269, 1989.

[14] R. Betarbet, T. B. Sherer, G. MacKenzie, M. Garcia-Osuna, A. V. Panov, and J. T. Greenamyre, "Chronic systemic pesticide exposure reproduces features of Parkinson's disease," Nature Neuroscience, vol. 3, no. 12, pp. 1301-1306, 2000.

[15] M. H. Polymeropoulos, C. Lavedan, E. Leroy et al., "Mutation in the alpha-synuclein gene identified in families with Parkinson's disease," Science, vol. 276, no. 5321, pp. 2045-2047, 1997.

[16] R. Krüger, W. Kuhn, T. Müller et al., "Ala30Pro mutation in the gene encoding alpha-synuclein in Parkinson's disease," Nature Genetics, vol. 18, no. 2, pp. 106-108, 1998.

[17] J. J. Zarranz, J. Alegre, J. C. Gómez-Esteban et al., “The new mutation, E46K, of alpha-synuclein causes Parkinson and lewy body dementia," Annals of Neurology, vol. 55, no. 2, pp. 164173, 2004

[18] M. C. Chartier-Harlin, J. Kachergus, C. Roumier et al., "Alphasynuclein locus duplication as a cause of familial Parkinson's disease," The Lancet, vol. 364, no. 9440, pp. 1167-1169, 2004.

[19] P. Ibáñez, A. M. Bonnet, B. Débarges et al., "Causal relation between alpha-synuclein gene duplication and familial Parkinson's disease," The Lancet, vol. 364, no. 9440, pp. 1169$1171,2004$.
[20] K. Nishioka, S. Hayashi, M. J. Farrer et al., "Clinical heterogeneity of alpha-synuclein gene duplication in Parkinson's disease," Annals of Neurology, vol. 59, no. 2, pp. 298-309, 2006.

[21] J. Fuchs, C. Nilsson, J. Kachergus et al., "Phenotypic variation in a large Swedish pedigree due to SNCA duplication and triplication," Neurology, vol. 68, no. 12, pp. 916-922, 2007.

[22] A. B. Singleton, M. Farrer, J. Johnston et al., "alpha-Synuclein locus triplication causes Parkinson 's disease," Science, vol. 302, no. 5646, p. 841, 2003.

[23] M. Farrer, J. Kachergus, L. Forno et al., "Comparison of kindreds with parkinsonism and alpha-synuclein genomic multiplications," Annals of Neurology, vol. 55, pp. 174-179, 2004.

[24] W. Dauer, N. Kholodilov, M. Vila et al., "Resistance of alphasynuclein null mice to the parkinsonian neurotoxin MPTP," Proceedings of the National Academy of Sciences of the United States of America, vol. 99, no. 22, pp. 14524-14529, 2002.

[25] V. Bonifati, P. Rizzu, F. Squitieri et al., "DJ-1 (PARK7), a novel gene for autosomal recessive, early onset parkinsonism," Neurological Sciences, vol. 24, no. 3, pp. 159-160, 2003.

[26] R. M. Canet-Avilés, M. A. Wilson, D. W. Miller et al., "The Parkinson's disease DJ-1 is neuroprotective due to cysteinesulfinic acid-driven mitochondrial localization," Proceedings of the National Academy of Sciences of the United States of America, vol. 101, no. 24, pp. 9103-9108, 2004.

[27] A. Mitsumoto and Y. Nakagawa, "DJ-1 is an indicator for endogenous reactive oxygen species elicited by endotoxin," Free Radical Research, vol. 35, no. 6, pp. 885-893, 2001.

[28] T. Taira, S. M. M. Iguchi-Ariga, and H. Ariga, "Co-localization with DJ-1 is essential for the androgen receptor to exert its transcription activity that has been impaired by androgen antagonists," Biological and Pharmaceutical Bulletin, vol. 27, no. 4, pp. 574-577, 2004.

[29] L. Zhang, M. Shimoji, B. Thomas et al., "Mitochondrial localization of the Parkinson's disease related protein DJ-1: implications for pathogenesis," Human Molecular Genetics, vol. 14, no. 14, pp. 2063-2073, 2005.

[30] E. Junn, W. H. Jang, X. Zhao, B. S. Jeong, and M. M. Mouradian, "Mitochondrial localization of DJ-1 leads to enhanced neuroprotection," Journal of Neuroscience Research, vol. 87, no. 1, pp. 123-129, 2009.

[31] R. H. Kim, P. D. Smith, H. Aleyasin et al., "Hypersensitivity of DJ-1-deficient mice to 1-methyl-4-phenyl-1,2,3,6- tetrahydropyrindine (MPTP) and oxidative stress," Proceedings of the National Academy of Sciences of the United States of America, vol. 102, no. 14, pp. 5215-5220, 2005.

[32] B. Thomas, R. von Coelln, A. S. Mandir et al., "MPTP and DSP-4 susceptibility of substantianigra and locus coeruleuscatecholaminergic neurons in mice is independent of parkin activity," Neurobiology of Disease, vol. 26, pp. 312-322, 2007.

[33] C. Wang, H. S. Ko, B. Thomas et al., "Stress-induced alterations in parkin solubility promote parkin aggregation and compromise parkin's protective function," Human Molecular Genetics, vol. 14, no. 24, pp. 3885-3897, 2005.

[34] N. Hattori, H. Matsumine, T. Kitada et al., "Molecular analysis of a novel ubiquitin-like protein (PARKIN) gene in Japanese families with AR-JP:, evidence of homozygous deletions in the PARKIN gene in affected individuals," Annals of Neurology, vol. 44, pp. 935-941, 1998.

[35] N. Abbas, C. B. Lücking, C. Ricard et al., "A wide variety of mutations in the parkin gene are responsible for autosomal 
recessive parkinsonism in Europe," Human Molecular Genetics, vol. 8, pp. 567-574, 1999.

[36] M. Kann, H. Jacobs, K. Mohrmann et al., "Role of parkin mutations in 111 community-based patients with early onset parkinsonism," Annals of Neurology, vol. 51, pp. 621-625, 2002.

[37] N. L. Khan, E. Graham, P. Critchley et al., "Parkin disease: a phenotypic study of a large case series," Brain, vol. 126, no. 6, pp. 1279-1292, 2003.

[38] K. Hedrich, C. Eskelson, B. Wilmot et al., "Distribution, type and origin of Parkin mutations: review and case studies," Movement Disorders, vol. 19, no. 10, pp. 1146-1157, 2004.

[39] E. M. Valente, A. R. Bentivoglio, P. H. Dixon et al., "Localization of a novel locus for autosomal recessive early-onset parkinsonism, PARK6, on human chromosome 1p35-p36," American Journal of Human Genetics, vol. 68, no. 4, pp. 895900, 2001.

[40] E. M. Valente, P. M. Abou-Sleiman, V. Caputo et al., "Hereditary early-onset Parkinson's disease caused by mutations in PINK1,” Science, vol. 304, no. 5674, pp. 1158-1160, 2004.

[41] Y. Hatano, Y. Li, K. Sato et al., "Novel PINK1 mutations in early-onset parkinsonism," Annals of Neurology, vol. 56, no. 3, pp. 424-427, 2004.

[42] D. G. Healy, P. M. Abou-Sleiman, J. M. Gibson et al., "PINK1 (PARK6) associated Parkinson disease in Ireland," Neurology, vol. 63, no. 8, pp. 1486-1488, 2004.

[43] C. F. Rohé, P. Montagna, G. Breedveld, P. Cortelli, B. A. Oostra, and V. Bonifati, "Homozygous PINK1 C-terminus mutation causing early-onset parkinsonism," Annals of Neurology, vol. 56, no. 3, pp. 427-431, 2004.

[44] Y. Li, H. Tomiyama, K. Sato et al., "Clinicogenetic study of PINK1 mutations in autosomal recessive early-onset parkinsonism," Neurology, vol. 64, no. 11, pp. 1955-1957, 2005.

[45] J. W. Pridgeon, J. A. Olzmann, L. S. Chin, and L. Li, "PINK1 protects against oxidative stress by phosphorylating mitochondrial chaperone TRAP1," PLoS Biology, vol. 5, no. 7, article e173, 2007.

[46] N. Exner, B. Treske, D. Paquet et al., "Loss-of-function of human PINK1 results in mitochondrial pathology and can be rescued by parkin," Journal of Neuroscience, vol. 27, no. 45, pp. 12413-12418, 2007.

[47] M. E. Haque, K. J. Thomas, C. D’Souza et al., "Cytoplasmic Pink1 activity protects neurons from dopaminergic neurotoxin MPTP," Proceedings of the National Academy of Sciences of the United States of America, vol. 105, no. 5, pp. 1716-1721, 2008.

[48] A. Wood-Kaczmar, S. Gandhi, Z. Yao et al., "PINK1 is necessary for long term survival and mitochondrial function in human dopaminergic neurons," PLoS ONE, vol. 3, no. 6, Article ID e2455, 2008.

[49] J. C. Greene, A. J. Whitworth, I. Kuo, L. A. Andrews, M. B. Feany, and L. J. Pallanck, "Mitochondrial pathology and apoptotic muscle degeneration in Drosophila parkin mutants," Proceedings of the National Academy of Sciences of the United States of America, vol. 100, no. 7, pp. 4078-4083, 2003.

[50] J. Park, S. B. Lee, S. Lee et al., "Mitochondrial dysfunction in Drosophila PINK1 mutants is complemented by parkin," Nature, vol. 441, no. 7097, pp. 1157-1161, 2006.

[51] I. E. Clark, M. W. Dodson, C. Jiang et al., "Drosophila pink1 is required for mitochondrial function and interacts genetically with parkin," Nature, vol. 441, no. 7097, pp. 1162-1166, 2006.
[52] C. T. Chu, "Tickled PINK1: mitochondrial homeostasis and autophagy in recessive Parkinsonism," Biochimica et Biophysica Acta, vol. 1802, no. 1, pp. 20-28, 2010.

[53] S. Geisler, K. M. Holmström, D. Skujat et al., "PINK1/Parkinmediated mitophagy is dependent on VDAC1 and p62/SQSTM1," Nature Cell Biology, vol. 12, no. 2, pp. 119-131, 2010.

[54] S. Kawajiri, S. Saiki, S. Sato et al., "PINK1 is recruited to mitochondria with parkin and associates with LC3 in mitophagy," FEBS Letters, vol. 584, no. 6, pp. 1073-1079, 2010.

[55] N. Matsuda, S. Sato, K. Shiba et al., "PINK1 stabilized by mitochondrial depolarization recruits Parkin to damaged mitochondria and activates latent Parkin for mitophagy," Journal of Cell Biology, vol. 189, no. 2, pp. 211-221, 2010.

[56] D. Narendra, A. Tanaka, D. F. Suen, and R. J. Youle, "Parkin is recruited selectively to impaired mitochondria and promotes their autophagy," Journal of Cell Biology, vol. 183, no. 5, pp. 795-803, 2008.

[57] D. P. Narendra, S. M. Jin, A. Tanaka et al., "PINK1 is selectively stabilized on impaired mitochondria to activate Parkin," PLoS Biology, vol. 8, no. 1, Article ID e1000298, 2010.

[58] C. Vives-Bauza, C. Zhou, Y. Huang et al., "PINK1-dependent recruitment of Parkin to mitochondria in mitophagy," Proceedings of the National Academy of Sciences of the United States of America, vol. 107, no. 1, pp. 378-383, 2010.

[59] A. Beilina, M. Van Der Brug, R. Ahmad et al., "Mutations in PTEN-induced putative kinase 1 associated with recessive parkinsonism have differential effects on protein stability," Proceedings of the National Academy of Sciences of the United States of America, vol. 102, no. 16, pp. 5703-5708, 2005.

[60] S. Takatori, G. Ito, and T. Iwatsubo, "Cytoplasmic localization and proteasomal degradation of N-terminally cleaved form of PINK1,” Neuroscience Letters, vol. 430, no. 1, pp. 13-17, 2008.

[61] Y. Kim, J. Park, S. Kim et al., "PINK1 controls mitochondrial localization of Parkin through direct phosphorylation," Biochemical and Biophysical Research Communications, vol. 377, no. 3, pp. 975-980, 2008.

[62] C. Zhou, Y. Huang, Y. Shao et al., "The kinase domain of mitochondrial PINK1 faces the cytoplasm," Proceedings of the National Academy of Sciences of the United States of America, vol. 105, no. 33, pp. 12022-12027, 2008.

[63] C. H. Sim, D. S. S. Lio, S. S. Mok et al., "C-terminal truncation and Parkinson's disease-associated mutations down-regulate the protein serine/threonine kinase activity of PTEN-induced kinase-1," Human Molecular Genetics, vol. 15, no. 21, pp. 3251-3262, 2006.

[64] Y. Yang, Y. Ouyang, L. Yang et al., "Pink1 regulates mitochondrial dynamics through interaction with the fission/fusion machinery," Proceedings of the National Academy of Sciences of the United States of America, vol. 105, no. 19, pp. 7070-7075, 2008.

[65] C. A. Gautier, T. Kitada, and J. Shen, "Loss of PINK1 causes mitochondrial functional defects and increased sensitivity to oxidative stress," Proceedings of the National Academy of Sciences of the United States of America, vol. 105, no. 32, pp. 11364-11369, 2008.

[66] T. Amo, S. Sato, S. Saiki et al., "Mitochondrial membrane potential decrease caused by loss of PINK1 is not due to proton leak, but to respiratory chain defects," Neurobiology of Disease, vol. 41, no. 1, pp. 111-118, 2011.

[67] M. Shamoto-Nagai, W. Maruyama, Y. Kato et al., "An inhibitor of mitochondrial complex I, rotenone, inactivates proteasome 
by oxidative modification and induces aggregation of oxidized proteins in SH-SY5Y cells," Journal of Neuroscience Research, vol. 74, no. 4, pp. 589-597, 2003.

[68] W. Dauer and S. Przedborski, "Parkinson's disease: mechanisms and models," Neuron, vol. 39, no. 6, pp. 889-909, 2003.

[69] V. Jackson-Lewis and S. Przedborski, "Protocol for the MPTP mouse model of Parkinson's disease," Nature Protocols, vol. 2, no. 1, pp. 141-151, 2007.

[70] J. Q. Trojanowski, "Rotenone neurotoxicity: a new window on environmental causes of Parkinson's disease and related brain amyloidoses," Experimental Neurology, vol. 179, no. 1, pp. 6-8, 2003. 


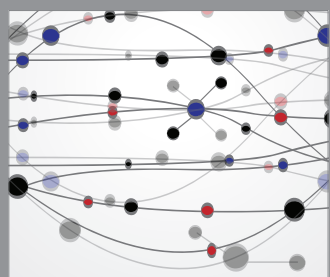

The Scientific World Journal
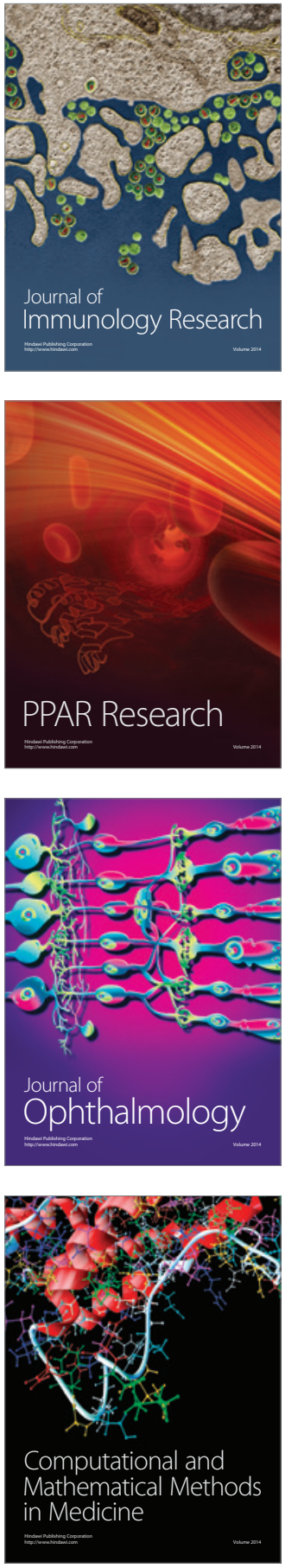

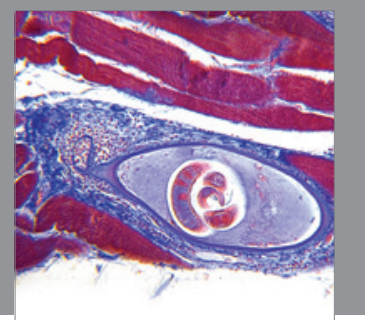

Gastroenterology

Research and Practice
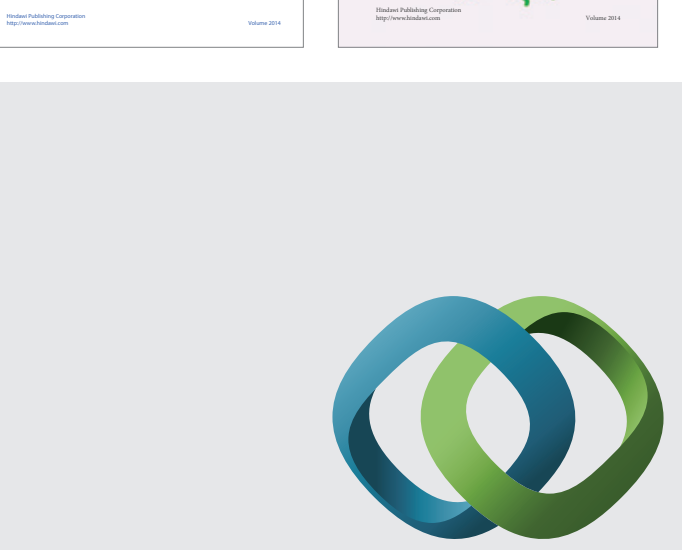

\section{Hindawi}

Submit your manuscripts at

http://www.hindawi.com
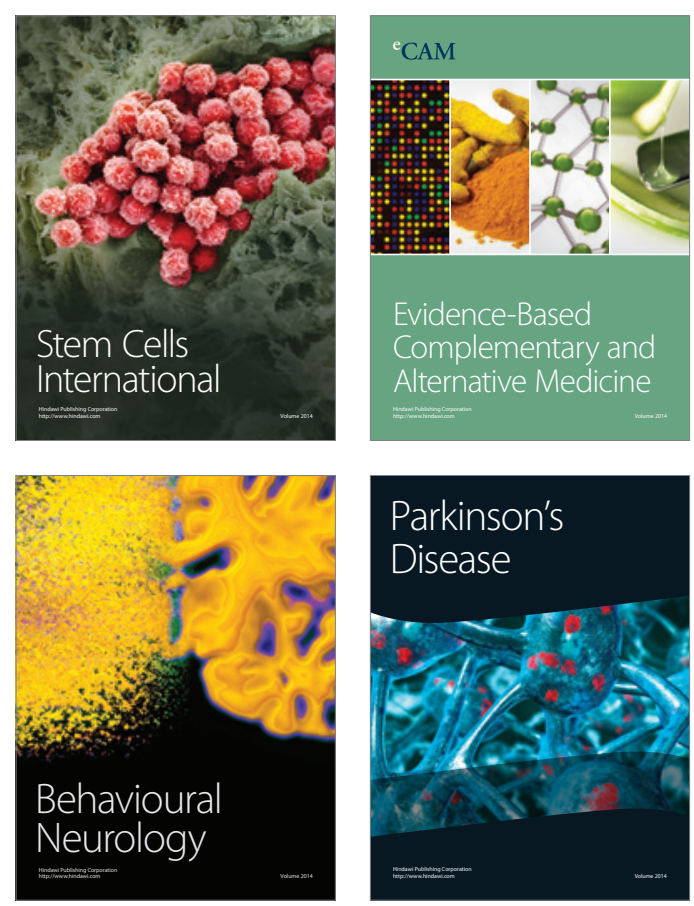

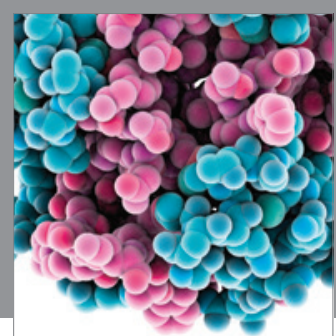

Journal of
Diabetes Research

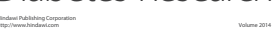

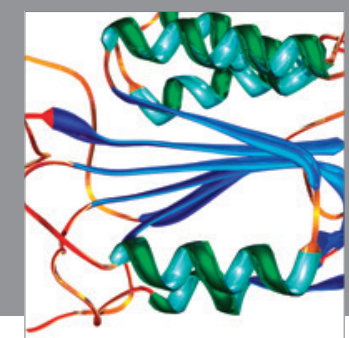

Disease Markers
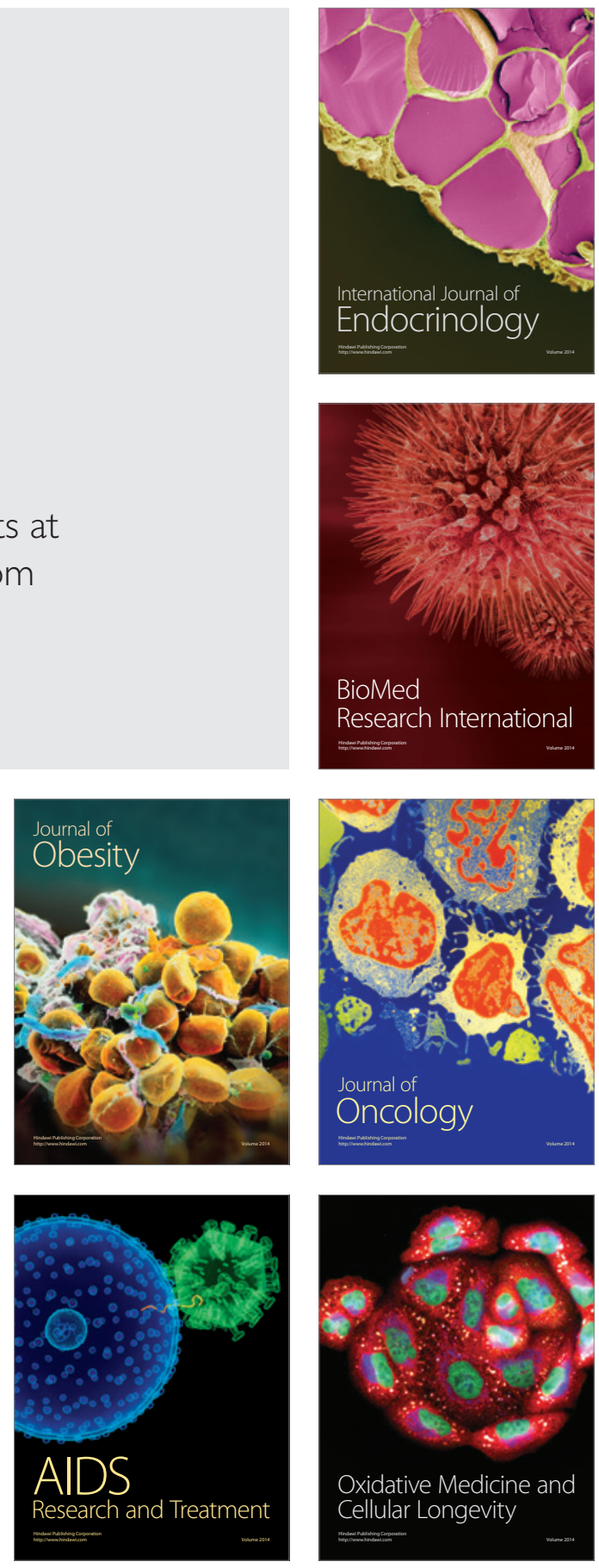